\title{
De-noising of an Image using Fuzzy Inference System and Performance Comparison with the Conventional system
}

\author{
Ahmed Farhan ${ }^{1}$, Rezwan us Saleheen ${ }^{2, *}$, Chen Li Wei ${ }^{1}$, Farhan Mahbub ${ }^{2}$ \\ ${ }^{1}$ College of Information and Communication Engineering, Harbin Engineering University, Heilongjiang, China. \\ ${ }^{2}$ Department of Mechatronics Engineering, World University of Bangladesh, Dhaka, Bangladesh.
}

Received: June 17, 2021, Revised: August 19, 2021, Accepted: August 31, 2021, Available Online: September 04,2021

\begin{abstract}
Noise prevailing in the image can diminish the physical appearance of the objects existing within the image and make them frail. Present research emphasizes a fuzzy inference system eradicating several types of noise from the images. The investigation implies the utilization of different levels of Salt \& Pepper noise. Followed by the pixel determination applying a mask, the disparity between the focused pixel's intensity with the minimum, average, and maximum power of the chosen window has been determined. Since two fuzzy valued outputs have been obtained to match them, the one provided by a low noise rate would demonstrate the more accurate filter for the selected window. Utilizing Matlab the Peak Signal-to-Noise ratio (PSNR) and Mean Square Error (MSE) are determined for evaluating the noise reduction performance. However, these values of PSNR and MSE obtained from this research are also compared with the conventional fuzzy filtering system.
\end{abstract}

Keywords: Fuzzy Logic, Fuzzy Inference System, Salt \& Pepper Noise, PSNR, MSE.

This work is licensed under a Creative Commons Attribution-Non Commercial 4.0 International License.

\section{Introduction}

The precision of processing an image debases with the existence of noise. So, de-noising of the image is notable in the arena of image processing. Nonlinear filtering procedures present superior results than linear techniques in the case of noise lessening. Current research on image processing using fuzzy logic shows that brilliant performance can be demonstrated using nonlinear approaches where fuzzy logic reasoning is the basis. Fuzzy logic deals with multiple values. However, Zadeh pioneered this logic. Computer software comprehends only binary functions. Conventional Boolean and Aristotelian logic contend with true or false, or the outright values of 0 and 1 . But, fuzzy logic expresses like medium, smaller, and higher. Everything in this world cannot always follow a linear function. Fuzzy logic considers these phenomena. A conventional set of binary logic deals with crisp values, and the fuzzy sets have fuzzy values. It contains linguistic variables. It tends to be characterized as low or small, average or medium, and big or high [1]. The values have fuzzy margins and can intersect each other [2].

FIS is established on fuzzy sets, rules, and reasoning [3]. Besides, fuzzy reasoning is estimated reasoning. This procedure draws assumptions from fuzzy rules and fuzzy sets. Fuzzifier, rule base, inference engine, and de-fuzzifier are the components of this procedure. In a fuzzifier, fuzzy sets are created from crisp values. Then, fuzzy rules are formed. The fuzzy rules to the fuzzy sets are applied through the inference engine. The fuzzy output is determined from this. The resultant is a fuzzy value. So, to get the crisp value as output, the de-fuzzification fuzzification process is needed [2].

\section{Literature Review}

In modern days, scientists have developed various types of image de-noising methods using fuzzy logic. Mohebbian et al. [4] have used a combination of adaptive Type-2 Fuzzy filters and a Fast-ICA to filter a set of low-dose images. Moreover, they have utilized five different phantoms to examine several effects of de-noising. Because of some pictures, it is not helpful to use the deep learning method. The main innovation of this research is to convert the shot noise distribution to salt and pepper. Also, they removed noise from mapped images using independent and fast component analysis.

Some researchers have suggested a system that uses fuzzy cognitive maps to reduce noise from images and mean filters [5]. This proposed method minimizes the data loss in the noise reduction process with the mean filter. Mahalakshmi and a group of researchers utilized an adaptive filter with the optimizationbased kernel interpolation and the type-2 fuzzy system to remove noise from the satellite image [6]. The authors have proposed three steps to remove noise from the images. They are noise identification, noise rectification, and enhancement of the image.

Golshan et al. [7] have developed an innovative Hysteresis Smoothing (HS) approach. Fuzzy norms are the basis of this method. In this method, fuzzy Hysteresis Smoothing is substituted by an interval soft manner. It allows the threshold levels to be determined equal to the fuzzy norm's free parameter. A. Saadia and A. Rashdi demonstrates an ultrasound image's denoising using a fuzzy weighted mean [8]. They have offered a fractional integration filter. A $3 * 3$ window is applied around distinct pixels. The windows are assigned using fuzzy logic. An adaptive fuzzy logic approach has been proposed by some scholars for speckle reduction of the ultrasound image. However, two levels have been used for adaptiveness. Fuzzy logic has been applied to the coefficients of variation calculated from the picture with speckle noise [9].

Ananthi and Balasubramaniam proposed an innovative impulse noise revealing technique based on fuzzy sets [10]. They offered to investigate image de-noising by modeling the nebulousness of image as entropy. The authors have implemented the minimization of entropy to generate an IVIFS for the testing image. An innovative fuzzy decision filter is 
projected in [11] to evade the misclassification of a rigid threshold in interchanging vector filters. A fuzzy membership value is imposed for rendering the possessions of pixels. Pixels are restricted between the original and noisy image [12]. When the innermost pixel is noise-free, the pixel keeps untouched. An innovative FDW-SVR de-noising algorithm has been illustrated by Zhang et al., assigning fuzzy precedence for every sample to its density weight [13].

Altogether the study focused on some specific outcomes. Noise is evacuated without any efforts to expressly distinguish it with the non-linear filters. Salt and pepper noise is also acknowledged as impulse or spike noise. The degraded image by impulse noise holds white pixels in the black areas and black pixels in the white sections [14]. Errors in A/D converters are the precursor of this type of noise [15]. This sort of noise is also acknowledged as data drop noise in light of its unique information dropping [16]. Spatial filters eradicate noise to a sensible degree yet at the expense of obscuring images which thus makes the edges in images undetectable. Therefore a diversity of the nonlinear median type filters, for example, relaxed and weighted median filters have been established to overcome this issue [17]. To afford a wider and appropriate understanding of Fuzzy logic, an undersized overview of fuzzy logic in different types of image de-noising approaches. An algorithm using minimum, average and maximum difference of intensity of the targeted pixel as input can obtain a fuzzy valued output. Hence comparing the two outcomes specifies the comparative values of noise and also a more approximate filter for the selected window.

\section{Methodology}

The image which is degraded by salt \& pepper noise includes dark pixels in the bright zones and bright pixels in the dark zones. The succeeding images are presented for visual comparison. Primarily, the original grayscale image has been shown and then the noisy image and the de-noised images by the projected method and the conventional fuzzy filtering method have been displayed for comparison. Fuzzy filters are grounded on 'IF and Then' rules. This is also known as fuzzy provisional statements. If ' $X$ ' denotes a universal set having ' $X$ ' number of elements and ' $A$ ' denotes a fuzzy set with the membership function $\mu_{\mathrm{A}}$, then

$$
A=\left\{\left(x, \mu_{A}(x)\right) \mid x \in X\right\} .
$$

The outcome of the fuzzy filters is governed by the fuzzy rules and the process of defuzzifying [18].

A real-life 'Leena' image [19] has been used as the original image, where different Salt \& Pepper noise levels have been added with the image to get the test image for the experiment. $1 \%$ of noise has been considered as low level and $10 \%$ as the high level. A mean filter is a linear filter that utilizes a mask over each pixel in the picture. In the experiment, every part of the pixels under the mask has gained an intermediate value to form a distinct pixel. This filter can remove little noise from the noisy image, which degrades the image's visual quality [20].

Like the mean filter, the median filter also degrades image quality and removes less noise, and removes essential information with the noise. These two filters have worked here as supportive filtering systems to remove some noise initially before applying the proposed method. Fuzzy Multilevel Median Filter is a median filter with multiple levels and is combined with fuzzy rules for eliminating impulse noise from the image. The resultant outcome is,

$$
\begin{aligned}
& r(x, y)=\operatorname{med}\left(\operatorname{med}_{\max }(x, y), \operatorname{med}_{\min }(x, y),\right. \\
& q(x, y))
\end{aligned}
$$

Here, $\operatorname{med}_{\mathrm{i}}(x, y)$ be the median values of the sub-windows $\mathrm{W}_{1}(x, y), \mathrm{W}_{2}(x, y), \mathrm{W}_{3}(x, y)$ and $\mathrm{W}_{4}(x, y)[18]$.

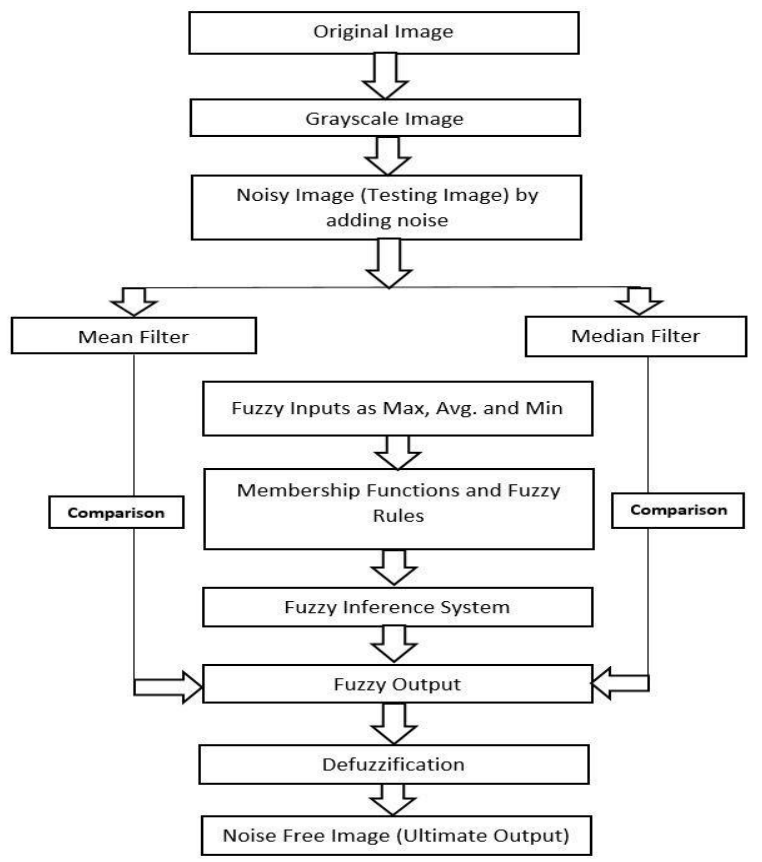

Fig. 1 Flow Chart of Proposed Fuzzy Inference System

The general system model is shown below in Fig. 1. Both mean and median filtering systems have been applied for the fuzzy inference system for comparison after getting the fuzzy inputs and outputs in fuzzification. Then, some membership functions have been formed for both inputs and output to fuzzify the data. After that, one $\mathrm{N} * \mathrm{~N}$ mask has been used to select the pixel, where $\mathrm{N}$ denotes an odd number where $\mathrm{N}>1$. It can be 3 , $5,7,9$, etc.

Then, the difference between the intensity of the targeted pixel with the minimum, average, and maximum intensity of the specific window has been determined. After that, the minimum, average, and maximum differences have been passed as the inputs and obtained a fuzzy valued output. Then, another output has been determined, and compared the two outcomes and checked the significant result. Moreover, it was checked whether the mean or median filter is more approximate for this selected window. The boundary pixel intensity interchanged with the average gray level value of the $\mathrm{N} * \mathrm{~N}$ mask with odd values (equal or greater than 3 ). This procedure was repeated to test all the pixels.

The fuzzy rules and the membership functions are the fundamental building block of the whole fuzzy inference system [21]. In the proposed method, three input variables having five membership functions have been used. Then, we used the outputs from the convolution of the mean and the median filter for comparison. The Mamdani model was used to make the fuzzy inference engine. This system will provide enhanced performance among all of the rules. Moreover, 125 fuzzy rules were estimated for the new assessment of the pixel under processing. 


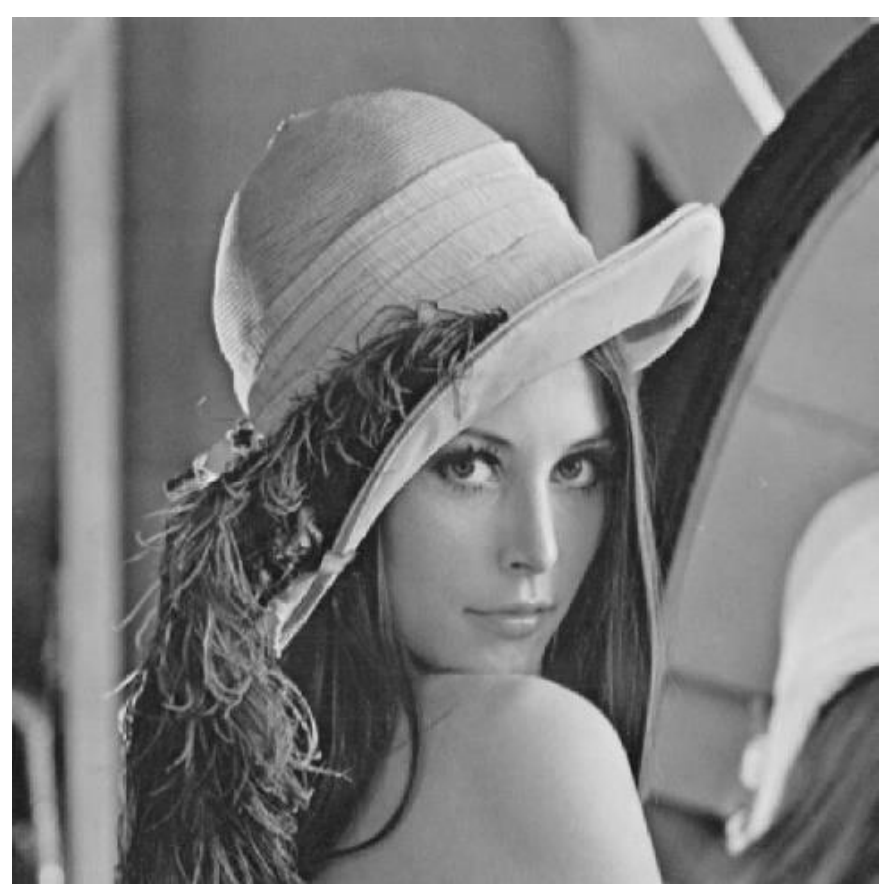

(a)

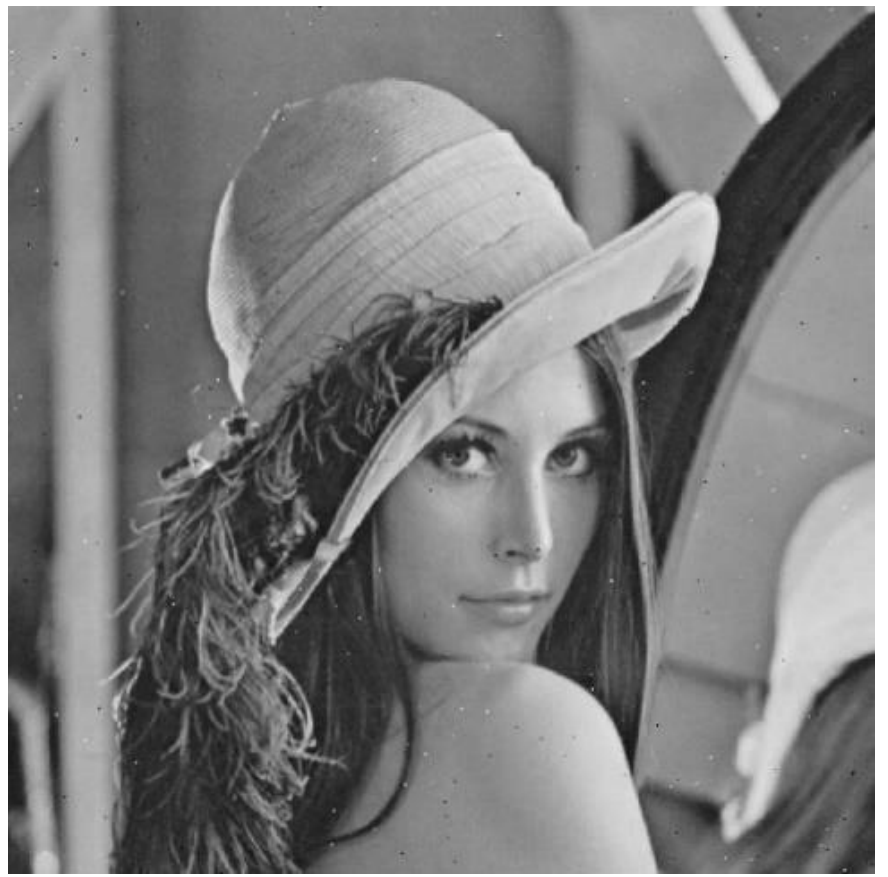

(c)

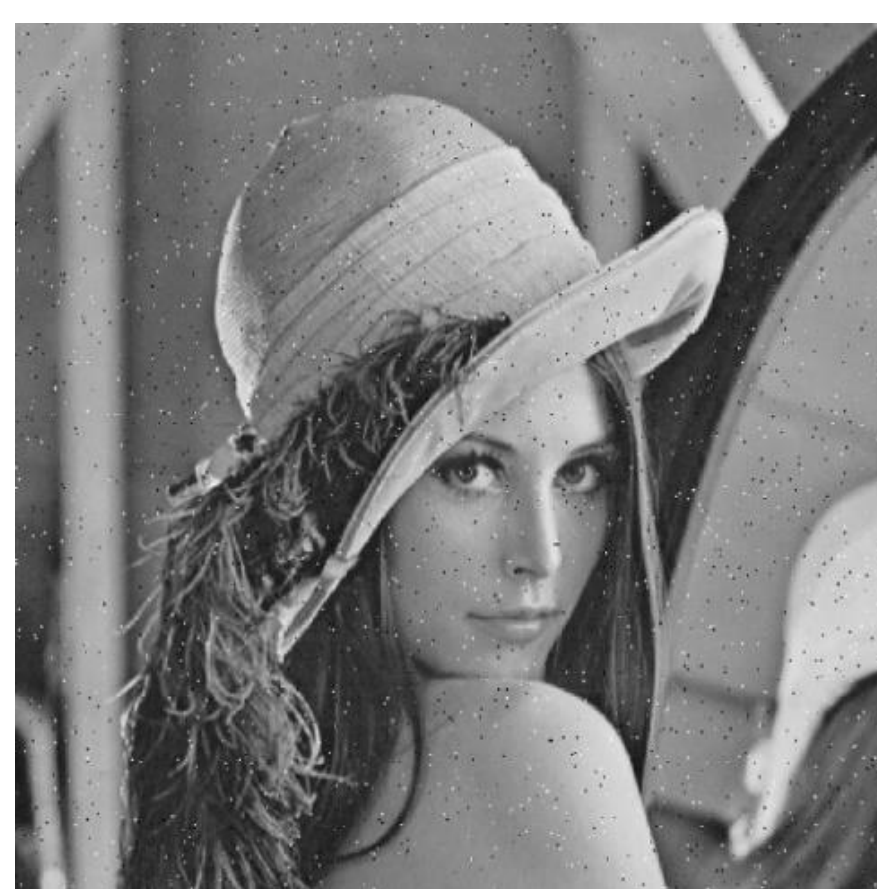

(b)

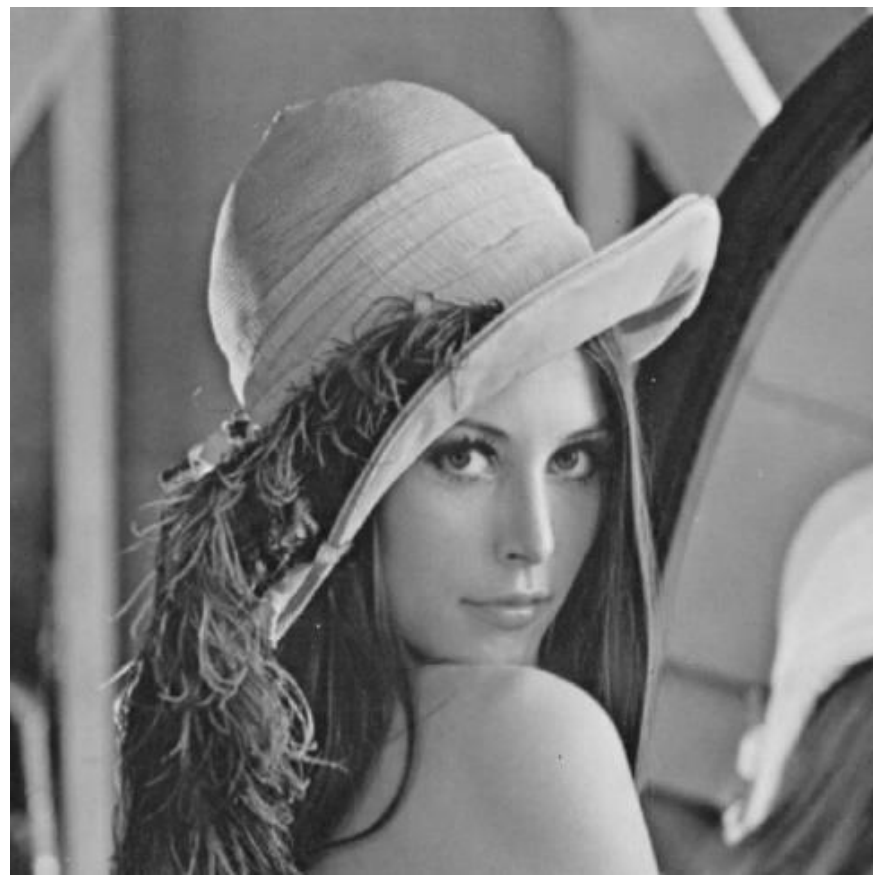

(d)

Fig. 2 (a) Original Gray Image, (b) Noisy Image (10\% of Salt \& Pepper noise), (c) Filtered image by the conventional method (PSNR is 31.0599, MSE is 50.9449), (d) Filtered image by our proposed method (PSNR is 31.6659, MSE is 44.2670).

Table 1 Performance comparison between conventional and our proposed method

\begin{tabular}{|c|c|c|c|c|}
\hline \multirow{2}{*}{$\begin{array}{c}\text { Noise } \\
\text { Level } \\
(\%)\end{array}$} & \multicolumn{2}{|c|}{ Peak Signal-to-Noise Ratio (PSNR) } & \multicolumn{2}{c|}{ Mean Square Erro (MSE) } \\
\cline { 2 - 5 } & $\begin{array}{c}\text { Conventional Fuzzy } \\
\text { Filtering System }\end{array}$ & Our Proposed Method & $\begin{array}{c}\text { Conventional Fuzzy } \\
\text { Filtering System }\end{array}$ & Our Proposed Method \\
\hline 1 & 32.6717 & 35.6879 & 35.1633 & 17.5421 \\
\hline 1.5 & 32.5148 & 35.3826 & 36.4113 & 18.8399 \\
\hline 2 & 32.3901 & 35.0967 & 37.5027 & 20.5159 \\
\hline 5 & 32.0311 & 33.6141 & 40.7449 & 28.3190 \\
\hline 10 & 31.0599 & 31.6659 & 50.9449 & 44.2670 \\
\hline
\end{tabular}


For the input variables, the membership functions are very small (VS), small (S), medium (M), large (L), and very large (VL). These are similar for both maximum and minimum inputs.

For the medium input variables, the membership functions are very small (VS), small (S), medium (M), large (L), and very large (VL). For inputs for average values, we also have used the Gaussian membership function.

For the output, five membership functions also have to be used for the system. But, these membership functions are not similar to the inputs, and their range is also different. We used triangular type membership functions for output.

Five membership functions for all the inputs have been used to make 125 fuzzy rules for our proposed system.

Pixel distance has been generated in the existing window. This distance of the pixels has been used to calculate the two values used to compare mean and median filtering techniques and decide which one is applicable for that window pixel. The membership functions of the inputs are used to generate fuzzy ifthen rules. Some of them are here,

- If the minimum distance is $\mathrm{S}$ and the median distance is VS, and the maximum distance is $\mathrm{S}$, then output is VL.

- If the minimum distance is $\mathrm{M}$ and median distance is $\mathrm{S}$, and the maximum distance is $\mathrm{M}$, then output is $\mathrm{L}$.

- If the minimum distance is $\mathrm{L}$ and the median distance is $\mathrm{L}$, and the maximum distance is VS, then output is $\mathrm{M}$.

- If the minimum distance is $\mathrm{L}$, and median distance is $\mathrm{L}$, and the maximum distance is $\mathrm{L}$, then output is $\mathrm{H}$.

- If the minimum distance is VL and the median distance is $\mathrm{S}$, and the maximum distance is $\mathrm{L}$, then output is $\mathrm{VH}$.

After getting the fuzzified result, de-fuzzification has been performed to acquire a crisp value. Several methods are used, like the center of the area $(\mathrm{CoA})$, the center of gravity $(\mathrm{CoG})$, and the mean of maximum methods. In our system, the most most commonly used defuzzification method centroid method or center of area method was used. This method determines the center of area of the fuzzy set and returns the corresponding crisp value. The defuzzification step translates this linguistic result into a numerical value.The surface viewer of our proposed fuzzy inference system has been used to calculate and get the crisp value.

The assessment of our recommended scheme by comparing it with the conventional fuzzy filtering method has been projected based on PSNR and MSE of the filtered images.

The fraction of the concentrated probable power of a signal and the noise that disturbs the dependability of its demonstration is considered as PSNR of that signal. It is mostly used for determining the feature of restoration of the image. It is frequently stated in terms of logarithmic decibel measurement.

$$
P S N R=10 \log _{10}\left(\frac{M a x_{I}^{2}}{M S E}\right)
$$

$\operatorname{Max}_{I}$ is the highest potential value of the pixel of an image. For pixels with 8 bits per sample, the value of $\operatorname{Max}_{I}$ is 255. PSNR can be calculated from the known value of MSE.

MSE is as well acknowledged as mean square deviation or MSD. It is the measurement of the feature of an estimator. It is a risk function. It is always positive and non-zero. It has the similar unit of dimension as the square of the magnitude is assessed like variance.

$$
\begin{aligned}
& M S E=\sqrt[2]{\frac{1}{M N}} \sum_{x=0}^{M-1} \sum_{y=0}^{N-1}[F(x, y)- \\
& f(x, y)]^{2}
\end{aligned}
$$

Here, $\mathrm{F}(\mathrm{x}, \mathrm{y})$ is the absolute resultant image obtained from the suggested method and $f(x, y)$ denotes the novel image. $M$ and $\mathrm{N}$ denote the height and the width of the testing grayscale image. If PSNR is known, then MSE can also be calculated from Eq. (3).

\section{Results Analysis and Discussion}

Fig. 2 represents the visual comparison of the noisy image and the output images for both methods. Table 1 shows the comparison of performance parameters of both methods.

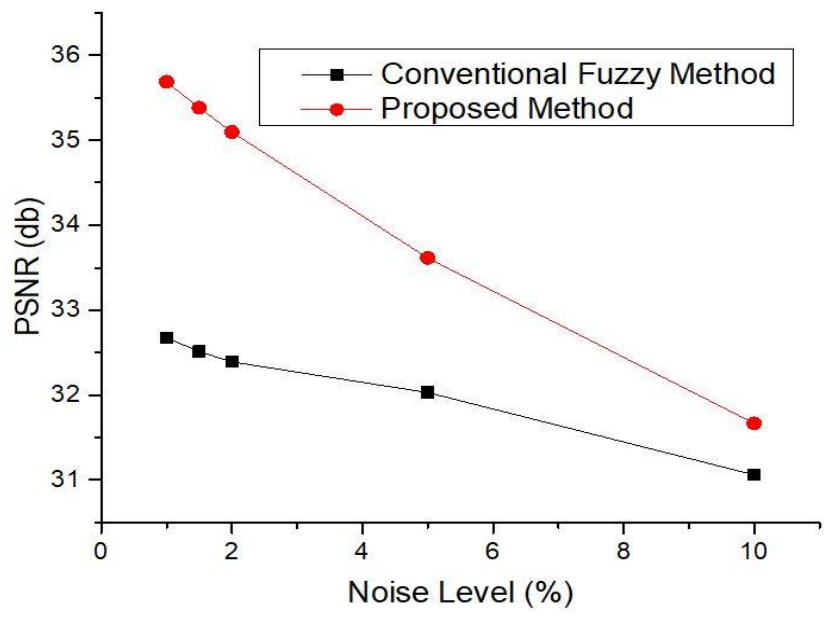

Fig. 3 PSNR versus Noise Level

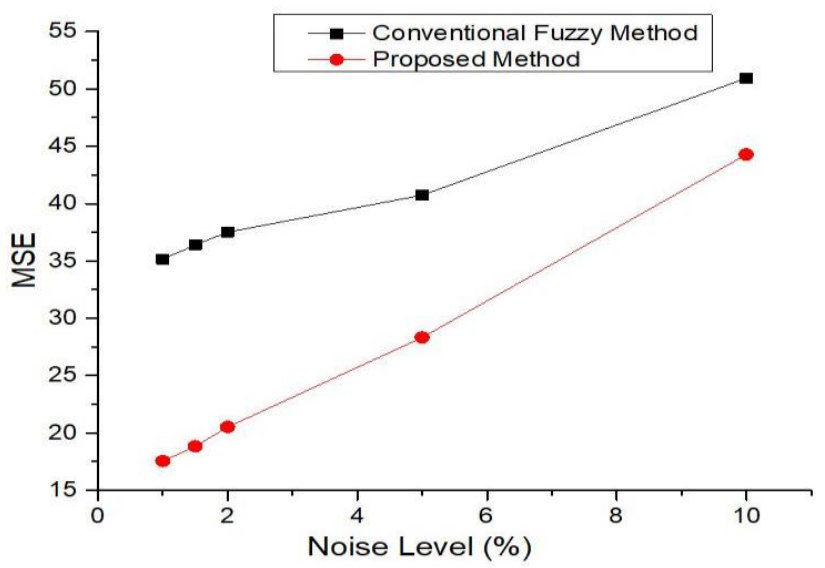

Fig. 4 MSE versus Noise Level

Fig. 3 and Fig. 4 represent the graphical comparisons of the performance parameters. The graphical representation in Fig. 3 is for different Speckle Noise level. $1 \%$ or 0.01 has been considered as the minimum noise level and $10 \%$ or 0.1 has been taken as a maximum noise level for this experiment. The method has been designed in such a way that the experiment can be directed for any level of noises (less or more) in the testing image. This graph demonstrates the change of the PSNR value with the growth of the noise level. With the rise of noise level, the value of PSNR drops for both cases. 
In the case of our recommended method, there is a significant change in the value of PSNR for noise level 0.01 to 0.2 . For the traditional method, there was no dramatic change in PSNR value. From the graphical display, we notice that the values of PSNR for all noise levels are better than the traditional fuzzy filtering system. Thus, the projected method can deliver a better result in the case of eliminating speckle noise.

The graph in Fig. 4 is for different Speckle Noise levels. 1\% or 0.01 has been considered as low noise level and $10 \%$ or 0.1 has been taken as a high noise level for this experiment. The structure has been planned in such a technique that the experimentation can be directed for any level of noises (less or more). This line-graph clarifies the rise of the MSE value with the growth of the noise level. That illustrates the reduction of error is optimum for our proposed method.

\section{Conclusion}

A novel approach based on a fuzzy inference system has been proposed to eradicate noise from a noisy image better than other filtering systems. The correlated works and the deficiencies of previous research works have been highlighted and evaluated here. The projected system had been generated dependent on the mean and median filter, fuzzy logic, and fuzzy inference system, where a noisy image has been used as the test image. The difference between the targeted pixel's minimum, average, and maximum window intensity was calculated and passed as input, and fuzzy valued outputs were obtained using the same procedure. We compared them to select the more appropriate filter for this chosen window and the outcomes. These outcomes and the fuzzy conventional filtering method's outcomes have been compared using PSNR and MSE as the comparison factors. The measurable experiments appear that our recommended procedure works admirably for the salt and pepper noise. Better PSNR denotes better effectiveness, where the shrink of MSE implies more minor errors.

The further recommendation is to add additional filters, for example, Gaussian smoothing, to compare extra output and find out the best filtering system for the contemporary window. Therefore, more data will be analyzed, and the efficiency will be superior to this model.

\section{References}

[1] Blanes-Vidal, V., Cantuaria, M.L. and Nadimi, E.S., 2017. A novel approach for exposure assessment in air pollution epidemiological studies using neuro-fuzzy inference systems: Comparison of exposure estimates and exposurehealth associations. Environmental research, 154, pp.196203.

[2] Godil, S.S., Shamim, M.S., Enam, S.A. and Qidwai, U., 2011. Fuzzy logic: A "simple" solution for complexities in neurosciences?. Surgical neurology international, 2.

[3] Farhan, A., Wei, C.L. and Ahmed, M.T., 2018. A Qualitative Overview of Fuzzy Logic in ECG Arrhythmia Classification. International Journal of Engineering Works, 5(11), pp. 232239.

[4] Mohebbian, M.R., Hassan, A.M., Wahid, K.A. and Babyn, P., 2020, June. Multi-Frame Low-Dose CT Image noise reduction using Adaptive Type-2 Fuzzy filter and Fast-ICA. In 2020 IEEE Region 10 Symposium (TENSYMP) (pp. 690693). IEEE.
[5] Altundogan, T.G. and Karakose, M., 2020, May. A Noise Reduction Approach Using Dynamic Fuzzy Cognitive Maps for Vehicle Traffic Camera Images. In 2020 Zooming Innovation in Consumer Technologies Conference (ZINC) (pp. 15-20). IEEE.

[6] Mahalakshmi, T. and Sreenivas, A., 2020. Adaptive Filter with Type-2 Fuzzy System and Optimization-Based Kernel Interpolation for Satellite Image Denoising. The Computer Journal, 63(6), pp.913-926.

[7] Golshan, H. and Hasanzadeh, R.P., 2021. Fuzzy Hysteresis Smoothing: A New Approach for Image Denoising. IEEE Transactions on Fuzzy Systems, 29(3), pp.686-697.

[8] Saadia, A. and Rashdi, A., 2016. Fractional order integration and fuzzy logic based filter for denoising of echocardiographic image. Computer methods and programs in biomedicine, 137, pp.65-75.

[9] Babu, J.J.J. and Sudha, G.F., 2016. Adaptive speckle reduction in ultrasound images using fuzzy logic on Coefficient of Variation. Biomedical Signal Processing and Control, 23, pp.93-103.

[10] Ananthi, V.P. and Balasubramaniam, P., 2016. A new image denoising method using interval-valued intuitionistic fuzzy sets for the removal of impulse noise. Signal Processing, 121, pp.81-93.

[11] Wang, G., Zhu, H. and Wang, Y., 2015. Fuzzy decision filter for color images denoising. Optik, 126(20), pp.2428-2432.

[12] Wang, G., Liu, Y., Xiong, W. and Li, Y., 2018. An improved non-local means filter for color image denoising. Optik, 173, pp.157-173.

[13] Zhang, Y., Xu, S., Chen, K., Liu, Z. and Chen, C.P., 2016. Fuzzy density weight-based support vector regression for image denoising. Information Sciences, 339, pp.175-188.

[14] Singh, V., Dev, R., Dhar, N.K., Agrawal, P. and Verma, N.K., 2018. Adaptive type-2 fuzzy approach for filtering salt and pepper noise in grayscale images. IEEE transactions on fuzzy systems, 26(5), pp.3170-3176.

[15] Farooque, M.A. and Rohankar, J.S., 2013. Survey on various noises and techniques for denoising the color image. International Journal of Application or Innovation in Engineering \& Management (IJAIEM), 2(11), pp.217-221.

[16] Boyat, A.K. and Joshi, B.K., 2015. A review paper: noise models in digital image processing. arXiv preprint arXiv:1505.03489.

[17] Motwani, M.C., Gadiya, M.C., Motwani, R.C. and Harris, F.C., 2004, September. Survey of image denoising techniques. In Proceedings of GSPX (Vol. 27, pp. 27-30). Proceedings of GSPX.

[18] Nachtegael, M., Van der Weken, D., Van De Ville, D. and Kerre, E.E. eds., 2013. Fuzzy filters for image processing (Vol. 122). Springer.

[19] Gonzalez, R.C. and Woods, R.E., 2008. Digital image processing: Pearson International Edition.

[20] Patidar, P., Gupta, M., Srivastava, S. and Nagawat, A.K., 2010. Image de-noising by various filters for different noise. International journal of computer applications, 9(4), pp.4550.

[21] Ahmad, M.T., Greenspan, M., Asif, M. and Marshall, J.A., 2018, April. Robust Apple Segmentation using Fuzzy Logic. In 2018 5th International Multi-Topic ICT Conference (IMTIC) (pp. 1-5). IEEE. 